\title{
Impact of telemedicine on the clinical outcomes and healthcare costs of patients with chronic heart failure and mid-range or preserved ejection fraction managed in a multidisciplinary chronic heart failure programme: $A$ sub-analysis of the iCOR randomized trial
}

Journal of Telemedicine and Telecare 0 (0) $1-9$

(C) The Author(s) 2018 Article reuse guidelines: sagepub.com/journals-permissions DOI: 10.1 I $77 / 1357633 \times 18796439$ journals.sagepub.com/home/jtt

@SAGE

\author{
Santiago Jiménez-Marrero ${ }^{1,2}$, Sergi Yun ${ }^{1,2,3}$, \\ Miguel Cainzos-Achirical,2,4, Cristina Enjuanes ${ }^{1,2}$, \\ Alberto Garay ${ }^{1,2}$, Nuria Farre ${ }^{5,6,7}$, Jose M Verdú ${ }^{7,8,9}$, \\ Anna Linas ${ }^{5,6}$, Pilar Ruiz ${ }^{5,6}$, Encarnación Hidalgo ${ }^{1,2}$, \\ Esther Calero ${ }^{1,2,9}$ and Josep Comín-Colet ${ }^{1,2,10}$
}

\begin{abstract}
Background: The efficacy of telemedicine in the management of patients with chronic heart failure and left ventricular ejection fraction $\geq 40 \%$ is poorly understood. The aim of our analysis was to evaluate the efficacy of a telemedicinebased intervention specifically in these patients, as compared to standard of care alone.

Methods: The Insuficiència Cardiaca Optimització Remota (iCOR) study was a single centre, randomised, controlled trial, designed to evaluate a telemedicine intervention added to an existing hospital/primary care multidisciplinary, integrated programme for chronic heart failure patients. 178 participants were randomised to telemedicine or usual care, and were followed for six months. For the present sub-analysis, only iCOR participants $(n=116)$ with left ventricular ejection fraction $\geq 40 \%$ were included. The primary study endpoint was the incidence of an acute nonfatal heart failure event, defined as a new episode of worsening of symptoms and signs consistent with acute heart failure requiring intravenous diuretic therapy. The healthcare-related costs in each study group were also evaluated.

Results: The incidence of the first occurrence of the primary endpoint was significantly lower in the telemedicine arm ( $22 \%$ vs $56 \%, p<0.001$ ), with a hazard ratio of 0.33 comparing to the usual care arm ( $95 \%$ confidence interval $0.17-0.64)$. Telemedicine was also associated with lower mean overall chronic heart failure care-related costs compared to usual care $(8163 €$ vs $4993 €, p=0.001)$. The results were consistent in both left ventricular ejection fraction of $40-49 \%$ and left ventricular ejection fraction $\geq 50 \%$ patients.

Conclusions: Our results suggest that telemedicine is a promising strategy for the management of chronic heart failure patients with left ventricular ejection fraction $\geq 40 \%$. These findings should be replicated in larger cohorts.
\end{abstract}

\footnotetext{
'Community Heart Failure Program, Bellvitge University Hospital, Spain ${ }^{2}$ Bellvitge Biomedical Research Institute (IDIBELL), L'Hospitalet de Llobregat, Spain

${ }^{3}$ Department of Internal Medicine, Bellvitge University Hospital, Spain ${ }^{4}$ Johns Hopkins Ciccarone Center for the Prevention of Heart Disease, Johns Hopkins Medical Institutions, USA

${ }^{5}$ Heart Failure Unit, Hospital del Mar, Spain

${ }^{6}$ Heart Diseases Biomedical Research Group (GREC), Hospital del Mar Biomedical Research Institute (IMIM), Spain

${ }^{7}$ Department of Medicine, Universitat Autònoma de Barcelona, Spain
}

\footnotetext{
${ }^{8}$ Sant Martí de Provençals Primary Care Center, Institut Catalá de la Salut, Spain

${ }^{9}$ Primary Care Research Institute Jordi Gol (IDIAP), Spain

${ }^{10}$ Department of Clinical Sciences, University of Barcelona, Spain

Corresponding author:

Josep Comin-Colet, Hospital Universitari de Bellvitge, Department of Cardiology, $19^{\text {th }}$ Floor, Feixa Llarga s/n, 08907 Hospitalet de Llobregat, Barcelona, Spain.

Email: jcomin@bellvitgehospital.cat
} 


\section{Keywords}

Chronic heart failure, heart failure, telemedicine

Date received: 28 June 2018; Date accepted: 2 August 2018

\section{Introduction}

Chronic heart failure (CHF) is a growing public health issue. ${ }^{1-3}$ In recent decades, population ageing and improvements in the treatment of most cardiovascular conditions have resulted in an increasing incidence and prevalence of CHF in most Western countries. ${ }^{1-3}$ The epidemiological importance, the burden caused by this health issue, and its high lethality, ${ }^{4}$ highlight the need for improved management approaches.

Approximately half of the patients with $\mathrm{CHF}$ have a left ventricular ejection fraction (LVEF) $<40 \%$ - the so called 'heart failure with reduced LVEF' (HFrEF). The pathophysiology and clinical characteristics of HFrEF are currently well established, and research efforts in recent decades have resulted in the development of a number of evidence-based therapies available for the treatment of these patients. ${ }^{7-10}$ On the other hand, the pathophysiology and therapeutic options for the remaining half of patients with $\mathrm{CHF}$ and LVEF $\geq 40 \%$ are not so well established, ${ }^{11-14}$ and further research is warranted in this patient population.

Telemedicine has become one of the key areas of development and research in CHF management in the last decade. ${ }^{15-20}$ Although the effectiveness of telemedicine in CHF has been controversial, recent pooled data suggest an overall beneficial effect in patients at higher risk of events. ${ }^{18}$ Specifically, both structured telephone support and non-invasive telemonitoring showed promise in reducing mortality, heart failure (HF) hospitalizations and improving quality of life. However, most of the studies published so far included mostly patients with HFrEF, therefore, the efficacy of telemedicine in the management specifically of patients with CHF and LVEF $\geq 40 \%$ is poorly understood. ${ }^{18,21}$

In the recent Insuficiència Cardiaca Optimització Remota (iCOR) study, ${ }^{17}$ a randomised, single-centre, controlled trial evaluating the efficacy of telemedicine in a cohort of $\mathrm{CHF}$ patients including both LVEF $<40 \%$ and $\geq 40 \%$, telemonitoring and teleinterventions based on bio-measure analysis, and videoconferences with patients or caregivers showed benefits in terms of non-fatal events and healthcare cost reduction. ${ }^{17}$ The aim of the present subanalysis is to evaluate the efficacy of the intervention specifically in the subgroup of participants with LVEF $\geq 40 \%$, including HF patients with both mid-range ejection fraction
(HFmrEF, defined as a LVEF $40-49 \%)^{5}$ as well as patients with preserved ejection fraction (HFpEF, $\mathrm{LVEF} \geq 50 \%)^{5}$

\section{Materials and methods}

\section{Study design, context and population}

Details on the methods of the iCOR trial have been described before. ${ }^{17}$ Briefly, this was a single centre, randomised, controlled, open-label trial, designed to evaluate the efficacy of adding telemedicine to an existing hospital/primary care integrated chronic management programme for patients with CHF. ${ }^{17}$ The study was carried out in a previously existing, specialised, multidisciplinary, nurse-based, hospital/primary care integrated CHF management programme. ${ }^{22}$

The study recruitment period took place between December 2010-October 2012, and patients were recruited after discharge for an acute HF decompensation requiring hospital admission in our centre. Inclusion criteria for the $\mathrm{iCOR}$ trial were: age of 18 years or older; presence of a diagnosis of CHF, defined by the presence of $\geq 3$ months of typical signs and symptoms, plus evidence of underlying structural heart disease or current hospital admission for acute decompensated HF needing intravenous diuretics; and being alive at hospital discharge. Exclusion criteria were being institutionalised, cognitive impairment without a caregiver, end-of-life care, planned cardiac invasive procedures, or planned haemodialysis. All iCOR participants provided written informed consent, the study protocol was approved by the institutional review board of the Hospital del Mar Research Institute (IMIM), and was registered on the website www.ClinicalTrials.gov (NCT01495078). ${ }^{17}$

For the present analysis, only iCOR participants with a baseline LVEF $\geq 40 \%$ were included. No further inclusion or exclusion criteria were implemented.

\section{Baseline data collection}

Baseline information including demographic characteristics, functional evaluation, medical history, physical examination, laboratory test results and other relevant clinical data was collected from each patient's medical history. All participants underwent a complete 
psychosocial, self-efficacy and health-related quality of life evaluation.

\section{Study endpoints, follow-up and event ascertainment}

The primary study endpoint was the development of an acute non-fatal HF event, defined as a new episode of worsening of symptoms and signs consistent with acute HF requiring intravenous diuretic therapy. This included both HF decompensations leading to a hospitalization, as well as decompensations managed in the daycare clinic.

As secondary study endpoints, the following events were assessed: incident HF hospitalizations, cardiovascular hospitalizations, non-cardiovascular hospitalizations, all-cause hospitalizations, all-cause death, cardiovascular death; as well as the combined endpoints of all-cause death or non-fatal HF events and all-cause death or HF hospitalization.

Additionally, we evaluated the healthcare-related costs in each study group, using actual cost data derived from the care of these patients (rather than estimations). Details on the cost calculations used in the iCOR study have been published before. ${ }^{17}$

All participants were followed for a period of six months. Information on the occurrence of the study endpoints was obtained by study investigators from the hospital and primary care electronic medical records and/or by direct interview of the patients.

\section{Study interventions}

The acute phase in-hospital management as well as the acute and chronic pharmacological management of the patients included in the iCOR trial were consistent across patients, and followed the protocols of the local CHF unit, which were based on the standards and recommendations published by the relevant scientific societies. ${ }^{5,6}$ Also, all patients were evaluated in an early, post-discharge visit occurring no later than seven days after hospital discharge.

With regards to the chronic, post-discharge followup of the patients included in the iCOR trial, participants were randomised to either 'usual care' or 'telemedicine'. 'Usual care' consisted of several prespecified, on-site, face-to-face, structured follow-up encounters, and patients were instructed to selfmonitor their bio-measures on a daily basis and contact the nurses in the event of any abnormality (e.g. weight gain, incipient decompensation signs). In the case of a mild decompensation, nurses performed diuretic dose adjustments, either themselves or with the support of CHF physician specialist.

On the other hand, 'telemedicine' consisted of the same number of pre-specified, structured follow-up encounters, but these were performed remotely rather than on site, using videoconference or audioconference. In these remote visits, the interventions were identical to those conducted in the usual care arm, including health education and pharmacological up-titration interventions ('teleintervention'). Patients were instructed to self-monitor their bio-measures on a daily basis and register this information together with any HF signs and symptoms in an user-friendly software, which transferred this data immediately to the HF unit ('telemonitoring'). Automated algorithms were created to allow for the automatic identification of decompensations on their very early stages using this information, and nurses reviewed this data together with any alerts generated by the system on a daily basis. In the event of an alert, nurses actively contacted the patient, and medication adjustments were performed, either by themselves or with the support of CHF physician specialist, following the same protocols as those used in the usual care arm.

\section{Statistical analyses}

Demographic and clinical baseline characteristics were summarised overall and by study arm. For continuous variables, the mean and standard deviation were calculated. For categorical variables, the number and \% were reported. Comparisons between study arms were performed using Student's $t$ and $\chi^{2}$ tests for continuous and categorical variables, respectively.

The incidence proportion of the first occurrence of each of the study endpoints was calculated for each study arm, and these compared using $\chi^{2}$ tests. Additionally, Kaplan-Meier cumulative survivor function curves were used to describe the event-free survival experience of the two study groups specifically for the primary study endpoint, and log-rank tests were used to compare the curves. The associations between telemedicine (compared to usual care) and each of the study endpoints were also evaluated using Cox proportional-hazards regression models.

Unitary direct hospital costs were compared between treatment groups using non-parametric tests (Kolmogorov-Smirnov test for two independent samples).

All analyses were conducted both for the overall study population (i.e. iCOR participants with LVEF $\geq 40 \%$ ), as well as stratifying by LVEF subgroup (HFmrEF and HFpEF). Formal interaction tests for the interaction term study arm $\times$ LVEF subgroup were also conducted for each of the study endpoints.

All significance tests and confidence intervals (CIs) were constructed with a type I error (alpha) level of $5 \%$. A $p$ value of 0.05 was used as the threshold for statistical significance. All analyses were conducted 
using SPSS version 18.0 (IBM, Armonk, New York, USA).

\section{Results}

\section{Study population}

The original iCOR study population included 178 participants, 97 of which were randomised to usual care and 81 to telemedicine. ${ }^{17}$ For the present sub-analysis, 62 patients with LVEF $<40 \%$ were excluded, yielding a total study population of 116 participants, 66 of which had been randomised to the usual care arm, and 50 to the telemedicine arm (Figure 1). With regards to LVEF subgroups, 27 participants had HFmrEF, and 89 had HFpEF.

\section{Baseline characteristics of the study participants}

The baseline characteristics of the participants included in the present analysis are summarised in Table 1. Overall, median age was 77 years, $47 \%$ were women, and $28 \%$ were considered frail patients. Mean LVEF was $58 \%$, and almost half of the patients had a New York Heart Association (NYHA) functional class of III or IV. The most frequent measured comorbidity was hypertension $(91 \%)$, followed by iron deficiency $(64 \%)$. The prevalence of anaemia, chronic kidney disease, diabetes mellitus and atrial fibrillation was also very high. In terms of pharmacological management, $98 \%$ participants were using diuretics, $80 \%$ were treated with betablockers, and 58\% with angiotensinconverting-enzyme inhibitors (ACEIs) or angiotensin receptor blockers (ARBs).

After randomization, both study arms (usual care, telemedicine) were well balanced in terms of their baseline characteristics.

\section{Primary study endpoint}

A total of 79 events of the primary endpoint happened during follow-up, 18 in the telemedicine arm (11 first events) and 61 in the usual 23 care arm (37 first events) (Table 2). The incidence proportion of the first occurrence of the primary endpoint was significantly lower in the telemedicine arm than in the usual care arm $(22 \%$ vs $56 \%, p<0.001$ ), with a hazard ratio (HR) of 0.33 comparing the telemedicine arm, to the usual care arm (95\% CI 0.17-0.64). The Kaplan-Meier cumulative incidence curves of the primary study endpoint for both study arms are presented in Figure 2(a) ( $p$ value of the Log Rank test $<0.001$ ).

\section{Secondary study endpoints}

There were statistically significant lower risks of HFrelated, cardiovascular-related, and all-cause

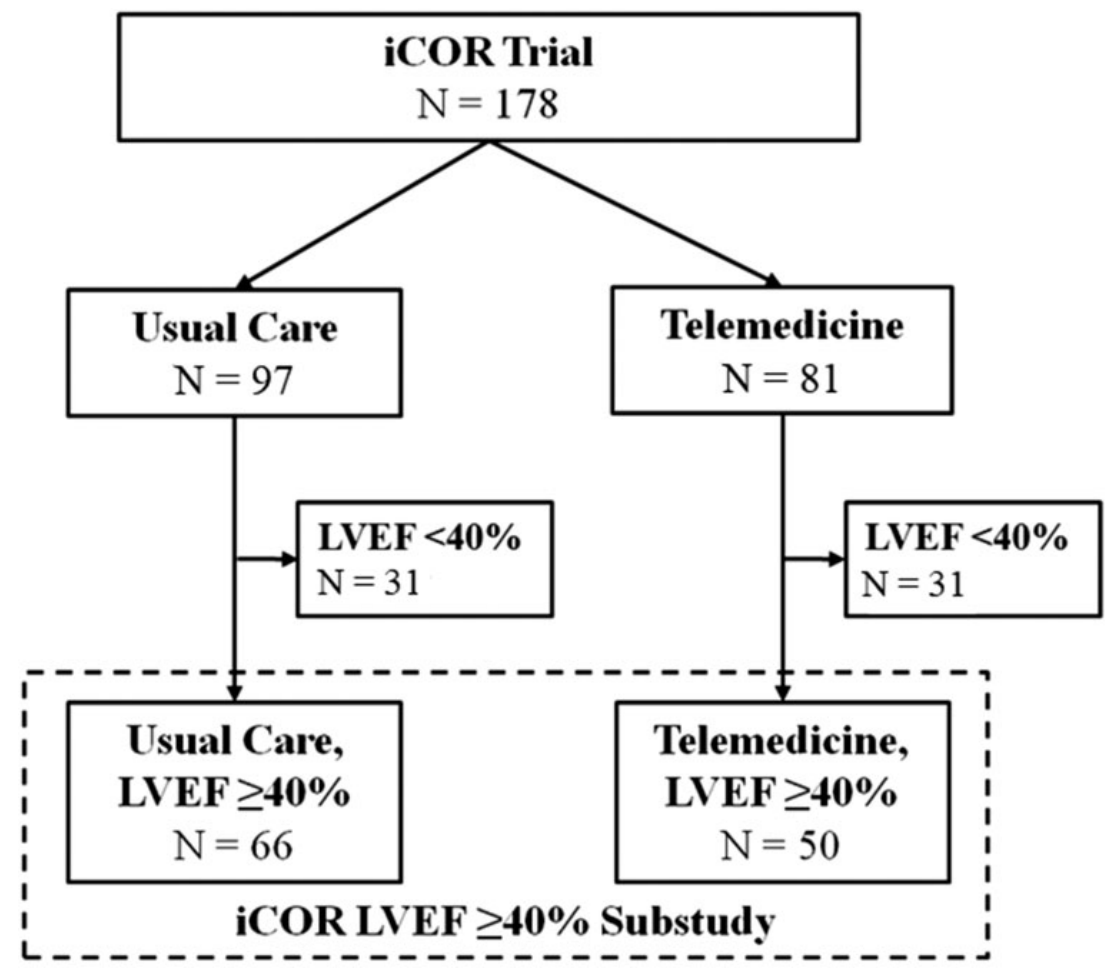

Figure I. Flowchart of the study participants.

iCOR: Insuficiència Cardiaca Optimització Remota; LVEF: left ventricular ejection fraction; N: number. 
Table I. Baseline characteristics of the study participants.

\begin{tabular}{|c|c|c|c|}
\hline & $\begin{array}{l}\text { Total } \\
(n=116)\end{array}$ & $\begin{array}{l}\text { Usual care } \\
(n=66)\end{array}$ & $\begin{array}{l}\text { Telemedicine } \\
(n=50)\end{array}$ \\
\hline Age, years & $77(10)$ & $78(10)$ & $77(\mathrm{II})$ \\
\hline Women & $55(47)$ & $32(49)$ & $23(46)$ \\
\hline BMI, $\mathrm{kg} / \mathrm{m}^{2}$ & $28(6)$ & $28(5)$ & $28(6)$ \\
\hline \multicolumn{4}{|l|}{ Blood pressure, $\mathrm{mm} \mathrm{Hg}$} \\
\hline Systolic & $124(22)$ & $126(19)$ & $122(25)$ \\
\hline Diastolic & $69(14)$ & $68(14)$ & $70(15)$ \\
\hline Heart rate, bpm & $73(13)$ & $74(13)$ & $71(12)$ \\
\hline \multicolumn{4}{|l|}{ NYHA functional class } \\
\hline $\mathrm{I}-\mathrm{II}$ & $60(52)$ & $36(55)$ & $24(48)$ \\
\hline III-IV & $56(48)$ & $30(46)$ & $26(52)$ \\
\hline LVEF, \% & $58(10)$ & $59(11)$ & $56(10)$ \\
\hline Ischaemic cause of HF & $37(32)$ & $21(32)$ & $16(32)$ \\
\hline \multicolumn{4}{|l|}{ Comorbidities } \\
\hline Hypertension & $106(9 \mid)$ & $61(92)$ & $45(90)$ \\
\hline Atrial fibrillation & $57(49)$ & $32(49)$ & $25(50)$ \\
\hline Diabetes mellitus & $58(50)$ & $34(52)$ & $24(48)$ \\
\hline $\mathrm{CKD}^{\mathrm{a}}$ & $71(6 I)$ & $41(62)$ & $30(60)$ \\
\hline COPD & $30(26)$ & $14(2 \mid)$ & $16(32)$ \\
\hline Iron deficiency ${ }^{\mathrm{b}}$ & $74(64)$ & $42(64)$ & $42(64)$ \\
\hline Anaemia & $65(56)$ & $38(58)$ & $27(54)$ \\
\hline \multicolumn{4}{|l|}{ Psychosocial evaluation } \\
\hline Self-efficacy, points & $21(10)$ & $20(10)$ & $21(10)$ \\
\hline Frailty & $33(28)$ & $17(26)$ & $16(32)$ \\
\hline \multicolumn{4}{|l|}{ Educational level } \\
\hline Illiterate & $6(5)$ & $3(5)$ & $3(6)$ \\
\hline Elementary education & $81(70)$ & $46(70)$ & $35(70)$ \\
\hline Middle school or higher & $29(25)$ & $17(26)$ & $12(24)$ \\
\hline \multicolumn{4}{|l|}{ Treatment } \\
\hline ACEI or ARBs & $67(58)$ & $38(58)$ & $29(58)$ \\
\hline Beta-blockers & $93(80)$ & $55(83)$ & $38(76)$ \\
\hline Aldosterone antagonist & $9(8)$ & $5(8)$ & $4(8)$ \\
\hline Digoxin & $23(20)$ & $14(2 \mid)$ & $9(18)$ \\
\hline Diuretics & I 14 (98) & $64(94)$ & $50(100)$ \\
\hline Hydralazine-nitrate combination & $32(28)$ & $17(25)$ & $15(39)$ \\
\hline Antiplatelet/anticoagulant therapy & $101(87)$ & $55(83)$ & $46(92)$ \\
\hline \multicolumn{4}{|l|}{ Laboratory measurements } \\
\hline Haemoglobin, g/dl & $12.1(2.8)$ & $11.9(3)$ & $12.4(2.4)$ \\
\hline $\mathrm{eGFR}, \mathrm{ml} / \mathrm{min} / \mathrm{l} .73 \mathrm{~m}^{2}$ & $58(26)$ & $56(25)$ & $60(27)$ \\
\hline NT-proBNP, pg/ml ${ }^{\mathrm{c}}$ & $1316(678-2792)$ & 1757 (7|8-345।) & $1056(649-2414)$ \\
\hline
\end{tabular}

ACEl: angiotensin-converting-enzyme inhibitor; ARB: angiotensin receptor blocker; BMI: body mass index; CKD: chronic kidney disease; COPD: chronic obstructive pulmonary disease; eGFR: estimated glomerular filtration rate; HF: heart failure; LVEF: left ventricular ejection fraction; NT-proBNP: N-terminal prohormone of brain natriuretic peptide; NYHA: New York Heart Association.

Data presented as number (\%) or mean (standard deviation), unless specified otherwise.

${ }^{\mathrm{a}}$ Defined as eGFR $<60 \mathrm{ml} / \mathrm{min} / \mathrm{l} .73 \mathrm{~m}^{2}$.

${ }^{b}$ Defined as ferritin $<100 \mathrm{n} / \mathrm{ml}$ or \% transferrin saturation (TSAT) $<20 \%$

'Presented as median and interquartile range.

hospitalizations comparing telemedicine to usual care (Table 2). On the other hand, hospitalization from noncardiovascular causes was similar in the two arms. With regards to fatal endpoints, the number of events was small in both arms, yielding wide $95 \%$ CIs that included the null value. Nevertheless, the incidence of all-cause death in the telemedicine arm $(6 \%)$ was half that in the usual care arm (12\%). Finally, telemedicine compared to usual care was also associated with a statistically significant lower risk of each of the combined study endpoints.

\section{Cost analyses}

In terms of healthcare costs, telemedicine was associated with significantly lower mean overall HF 
Table 2. Associations between study arms and incidence of each of the study endpoints.

\begin{tabular}{|c|c|c|c|c|c|c|c|c|c|}
\hline & \multicolumn{3}{|c|}{ Usual care } & \multicolumn{3}{|c|}{ Telemedicine } & \multirow[b]{2}{*}{$p$ Value $^{\mathrm{a}}$} & \multirow[b]{2}{*}{$\begin{array}{l}\mathrm{HR} \\
(95 \% \mathrm{Cl})\end{array}$} & \multirow[b]{2}{*}{$\begin{array}{l}p \text { Value } \\
\text { interaction }\end{array}$} \\
\hline & $\begin{array}{l}\text { No. } \\
\text { events }\end{array}$ & $\begin{array}{l}\text { No. Ist } \\
\text { events }\end{array}$ & $\begin{array}{l}\text { Incidence } \\
\text { Ist events } \\
(\%)\end{array}$ & $\begin{array}{l}\text { No. } \\
\text { events }\end{array}$ & $\begin{array}{l}\text { No. } \\
\text { Ist events }\end{array}$ & $\begin{array}{l}\text { Incidence } \\
\text { Ist events } \\
(\%)\end{array}$ & & & \\
\hline \multicolumn{10}{|l|}{ Primary study endpoint } \\
\hline Non-fatal HF events & 61 & 37 & 56 & 18 & 11 & 22 & $<0.001$ & $0.33(0.17-0.64)$ & 0.809 \\
\hline \multicolumn{10}{|l|}{ Secondary study endpoints } \\
\hline HF hospitalization & 29 & 23 & 35 & 10 & 7 & 14 & 0.011 & $0.38(0.16-0.90)$ & 0.933 \\
\hline CV hospitalization & 37 & 27 & 41 & 13 & 9 & 18 & 0.009 & $0.40(0.19-0.86)$ & 0.305 \\
\hline Non-CV hospitalization & 12 & 9 & 14 & 7 & 6 & 12 & 0.796 & $1.01(0.35-2.88)$ & 0.248 \\
\hline All-cause hospitalization & 51 & 33 & 50 & 21 & 14 & 28 & 0.017 & $0.52(0.28-0.98)$ & 0.218 \\
\hline All-cause death & 8 & 8 & 12 & 3 & 3 & 6 & 0.267 & $0.65(0.16-2.61)$ & 0.890 \\
\hline CV death & 6 & 6 & 9 & 2 & 2 & 4 & 0.286 & $0.66(0.12-3.58)$ & 0.566 \\
\hline $\begin{array}{l}\text { All cause death or } \\
\text { non-fatal HF event }\end{array}$ & 37 & 37 & 56 & $\mathrm{II}$ & II & 22 & $<0.001$ & $0.33(0.17-0.64)$ & 0.809 \\
\hline $\begin{array}{l}\text { All cause death of } \\
\text { HF hospitalization }\end{array}$ & 24 & 24 & 36 & 7 & 7 & 14 & 0.007 & $0.36(0.16-0.85)$ & 0.933 \\
\hline
\end{tabular}

$\mathrm{Cl}$ : confidence interval; CV: cardiovascular; HF: heart failure; HR: hazard ratio; No.: number.

Data are presented as number, incidence proportion (in \%), and as hazard ratios (and 95\% Cls) from the Cox Proportional Hazards regression models, respectively.

${ }^{a}$ Comparing the incidence proportion of each endpoint in the usual care arm and the telemedicine arm.

balue of $p$ for the interaction interaction term study arm*LVEF subgroup, for each study endpoint.

care-related costs compared to usual care (8163€ vs $4993 €, p=0.001)$. Regarding individual cost components, compared to telemedicine, usual care was associated with higher costs related to hospitalization (6064€ vs $2750 €, p=0.006$ ), and slightly higher costs related to diagnostic procedures (1386€ vs $576 €$, $p=0.001)$. On the other hand, telemedicine was associated with slightly higher costs related to ambulatory care $(712 €$ vs $1667 €, p=0.001)$.

\section{Subgroup analyses: HFmrEF and HFpEF}

The analyses for the primary and secondary study endpoints were also conducted further stratifying by LVEF $\geq 40 \%$ subtype (HFmrEF, HFpEF). The results of these analyses were consistent with those from the overall analyses, with similar benefits of telemedicine in patients with HFpEF and with HFmrEF (Table 2 and Figure 2(b)). Consistent with this, the $p$ values of all tests evaluating the interaction between study arms and LVEF subgroups were not statistically significant for any of the study endpoints.

\section{Discussion}

In this sub-analysis of a randomised controlled trial restricted to patients with $\mathrm{CHF}$ and $\mathrm{LVEF} \geq 40 \%$, telemedicine, as compared to usual care, was associated with a lower risk of non-fatal acute HF events, and a lower risk of hospitalization at six months of followup. These results were robust, and were consistent across LVEF subtypes (HFpEF, HFmrEF). Telemedicine may thus provide an opportunity to improve the outcomes of individuals with $\mathrm{CHF}$ and LVEF $\geq 40 \%$, a patient population in whom most pharmacological interventions have shown limited benefit. ${ }^{21}$ To our knowledge, this is the first study to specifically assess the potential benefits of telemedicine interventions in patients with CHF and LVEF $\geq 40 \%,{ }^{18,21}$ and has important implications for the management of these patients.

Potential mechanisms for the observed associations follow. First, despite the implementation in our area of a multidisciplinary, comprehensive, specialised, transitional chronic care programme including early postdischarge assessments, pre-specified visits and close, structured follow-up, ${ }^{22}$ it must be noted that between usual care visits, the patient is responsible for recording their HF signs, symptoms and bio-measures; and more important, for interpreting them and evaluating their implications (the nurse/physician only become aware of abnormalities between visits if the patient considers them important enough to contact the HF team). Although in our programme, CHF patients are systematically empowered with health education and detailed instructions on how to proceed in the event of a decompensation, unfortunately sometimes they fail to implement these recommendations, leading to severe decompensations requiring intravenous drugs and/or urgent hospitalization. In this context, telemedicine, via remote daily monitoring of the patient by the HF 

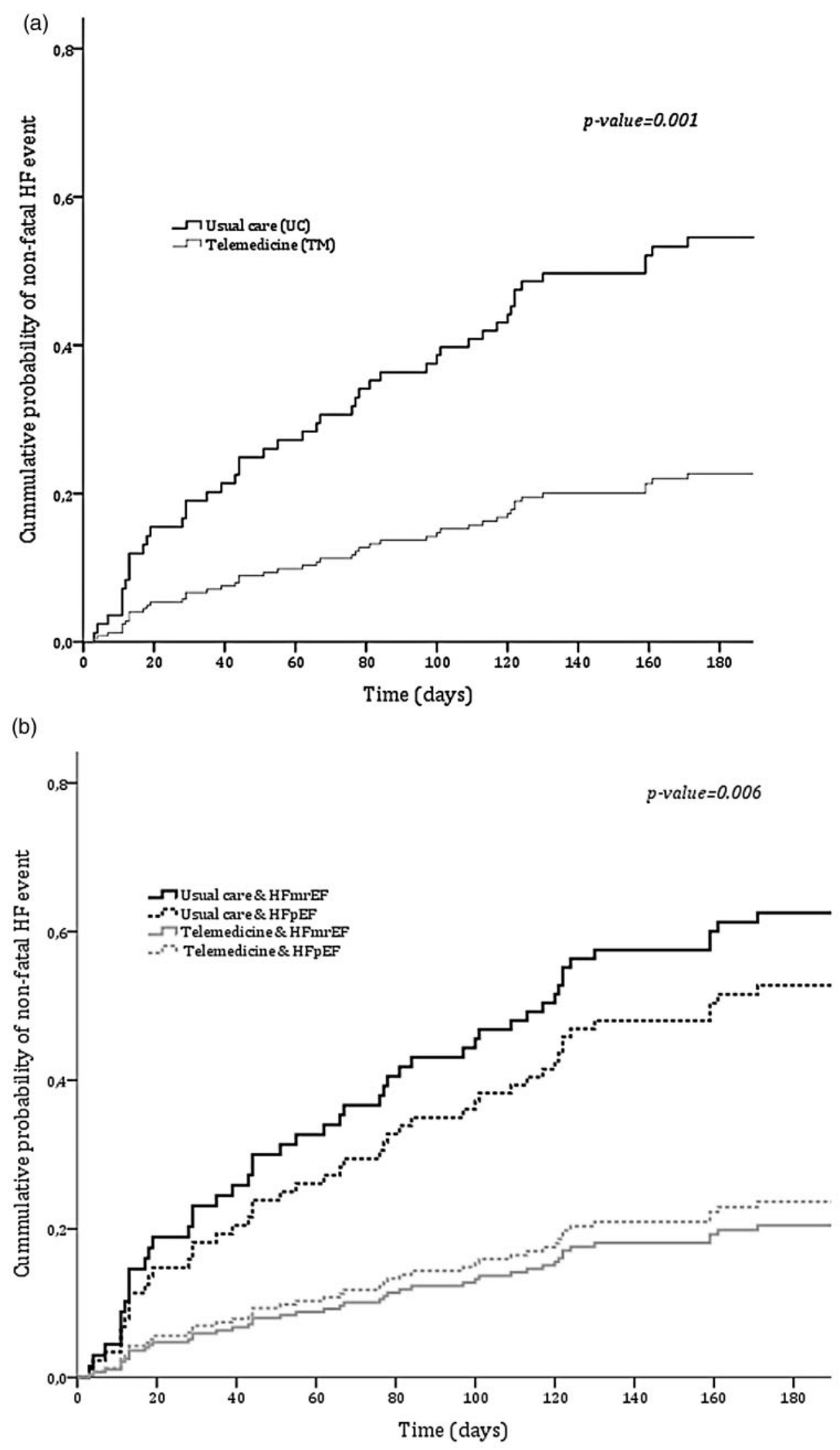

Figure 2. Cumulative incidence of the primary study endpoint, by study arm (panel (a)), and further stratified by left ventricular ejection fraction (LVEF) subtype (panel (b)).

HF: heart failure; HFmrEF: heart failure with mid-range ejection fraction; HFpEF: heart failure with preserved ejection fraction.

team allows for reminding the patient to record this information on a daily basis, as well as for the systematic evaluation of this data using automated algorithms, and for the early detection of mild $\mathrm{HF}$ decompensations. This allows treatment adjustments on a timely manner, preventing the further worsening of the patient and the need for intravenous drugs. Second, acute decompensations are frequent in patients with $\mathrm{CHF}$ and $\mathrm{LVEF} \geq 40 \%$. ${ }^{21}$ This was also the case in our study population, despite the multidisciplinary 
CHF programme to which all patients were exposed. The very high rates of events observed likely explain the fact that we were able to find statistically significant differences between the two study arms in spite of the short follow-up used and the small study population included.

Our study has important clinical implications. As of now, most trials involving pharmacological interventions have failed to show benefit in patients with $\mathrm{CHF}$ and LVEF $\geq 40 \% .{ }^{11-14}$ Thus, interventions providing a clinical benefit are particularly warranted in this group of patients, and are likely to have a large impact on their health outcomes. Importantly, in our study telemedicine not only showed an improvement in non-fatal outcomes, but also was associated with reduced healthcare costs, mostly due to a reduction in hospitalization-related costs. Although a formal costeffectiveness evaluation was not conducted, our results suggest thus that the improvements in clinical outcomes with telemedicine would not mean increased costs, but actually the opposite is true. With regards to the feasibility of the intervention and the acceptability of the technology used, previous studies conducted in Catalonia have shown that CHF patients have a high level of adherence to devices similar to those used in our intervention, resulting in a very low proportion of missed biometric daily transmissions. ${ }^{16,17}$ This supports the applicability of this technology in clinical practice and beyond the setting of a randomised controlled trial. Importantly, the same may not apply to other types of technology, the heterogeneity of which may explain the inconsistent results across previous studies evaluating different types of telemedicine interventions in patients with $\mathrm{CHF} .{ }^{20}$ If replicated in larger cohorts, our findings suggest that telemedicine, implemented using a technology similar to that used in our study, should be considered for its incorporation as part of standard $\mathrm{CHF}$ management programmes, including those involving patients with LVEF $\geq 40 \%$.

\section{Study limitations}

Our study has some limitations, mostly derived from the single-centre nature of the trial and from the posthoc, subgroup nature of the present analysis. First, because of the small sample size and the short followup period, the number of deaths was relatively small, and we were not sufficiently powered to identify differences between the study arms in terms of fatal endpoints. However, the incidence of all-cause death in the usual care arm was twice that in the telemedicine arm, pointing to a potential benefit of the intervention also in terms of this endpoint. Larger studies are needed to better understand the potential benefits of telemedicine in terms of death in these patients.
Second, the small sample size also likely limited the statistical power of the interaction tests. However, a priori we have no reasons to expect telemedicine being less effective in patients with HFmrEF (which was the smallest study LVEF subgroup) than in patients with HFpEF. Again, studies with larger sample sizes are needed to better understand these potential nuances, specifically to assess patients with HFmrEF, a subgroup recently defined in the european clinical guidelines. ${ }^{5}$

Third, the cost analyses conducted were exploratory, the data available for the calculations was limited, and information on the costs of the programme per se was not available. Also, patient satisfaction and other components relevant to patients and users were not incorporated in the analysis. For all these reasons, these findings should be considered hypothesis-generating, and should be interpreted cautiously.

Fourth, the generalisability of the study findings to other centres and healthcare environments is currently unknown. Nevertheless, because in our area the usual care arm involved gold-standard chronic care model procedures, ${ }^{5,6,22}$ telemedicine compared to usual care may have even greater benefits in areas in which usual care is less developed.

Finally, the present results are consistent with those already published by our group as part of the main iCOR trial analysis. ${ }^{17}$ Nevertheless, the study included a non-negligible number of patients with $\mathrm{LVEF}<40 \%$, and the relatively small sample size would have limited the statistical power of any potential interaction tests. Therefore, we decided to focus our attention specifically on the subgroup of patients with CHF and $\mathrm{LVEF} \geq 40 \%$, in whom interventions that are effective in patients with $\mathrm{LVEF}<40 \%$ often fail to show a benefit; and for whom the relevant scientific societies have made calls for further research aimed at improving their health outcomes. ${ }^{5}$

\section{Conclusions}

In this pilot, single-centre, randomised controlled trial including CHF patients with $\mathrm{LVEF} \geq 40 \%$, telemedicine, as compared to usual care, was associated with a lower risk of non-fatal acute HF events and of hospitalization after six months of follow-up. If replicated in larger cohorts, these results suggest that telemedicine could represent a promising management approach to improve the health outcomes of patients with CHF and $\mathrm{LVEF} \geq 40 \%$, a patient population that is expected to grow in the coming decades, and for whom most pharmacological interventions have so far failed to show benefit. 


\section{Declaration of Conflicting Interests}

The authors declared the following potential conflicts of interest with respect to the research, authorship, and/or publication of this article: All authors listed above take responsibility for all aspects of the reliability and freedom from bias of the data presented and their discussed interpretation.

\section{Funding}

The authors received no financial support for the research, authorship and/or publication of this article.

\section{References}

1. Benjamin EJ, Blaha MJ, Chiuve SE, et al. American Heart Association Statistics Committee and Stroke Statistics Subcommittee. Heart disease and stroke statistics-2017 update: A report from the American Heart Association. Circulation 2017; 135: e146-e603.

2. Khera R, Pandey A, Ayers CR, et al. Contemporary epidemiology of heart failure in fee-for-service medicare beneficiaries across healthcare settings. Circ Hear Fail 2017; 10: 1-9. DOI: 10.1161/CIRCHEART FAILURE.117.004402

3. Ponikowski P, Anker SD, AlHabib KF, et al. Heart failure: Preventing disease and death worldwide. ESC Heart Failure 2014; 1: 4-25.

4. Bhatia RS, Tu JV, Lee DS, et al. Outcome of heart failure with preserved ejection fraction in a population-based study. $N$ Engl J Med 2006; 355: 260-269.

5. Ponikowski P, Voors AA, Anker SD, et al. 2016 ESC Guidelines for the diagnosis and treatment of acute and chronic heart failure: The Task Force for the diagnosis and treatment of acute and chronic heart failure of the European Society of Cardiology (ESC) Developed with the special contribution of the Heart Failure Association (HFA) of the ESC. Eur Heart J 2016; 37: 2129-2200.

6. Yancy CW, Jessup M, Bozkurt B, et al. 2017 ACC/AHA/ HFSA focused update of the 2013 ACCF/AHA guideline for the management of heart failure: A report of the American College of Cardiology/American Heart Association Task Force on Clinical Practice Guidelines and the Heart Failure Society of America. Circulation 2017; 136: e137-e161.

7. Yusuf S, Pitt B, Davis CE, et al. Effect of enalapril on survival in patients with reduced left ventricular ejection fractions and congestive heart failure. $N \mathrm{Engl} \mathrm{J} \mathrm{Med}$ 1991; 325: 293-302.

8. Packer M, Bristow MR, Cohn JN, et al. The effect of carvedilol on morbidity and mortality in patients with chronic heart failure. U.S. Carvedilol Heart Failure Study Group. N Engl J Med 1996; 334: 1349-1355.

9. Pitt B, Zannad F, Remme WJ, et al. J. The effect of spironolactone on morbidity and mortality in patients with severe heart failure. Randomized Aldactone Evaluation Study Investigators. N Engl J Med 1999; 341: 709-717.
10. McMurray JJ, Packer M, Desai AS, et al.; PARADIGM$\mathrm{HF}$ Investigators and Committees. Angiotensin-neprilysin inhibition versus enalapril in heart failure. $N$ Engl $J$ Med 2014; 371: 993-1004.

11. Yusuf S, Pfeffer MA, Swedberg K, et al.; CHARM Investigators and Committees. Effects of candesartan in patients with chronic heart failure and preserved leftventricular ejection fraction: The CHARM-Preserved Trial. Lancet 2003; 362: 777-781.

12. Cleland JG, Tendera M, Adamus J, et al.; PEP-CHF Investigators. The perindopril in elderly people with chronic heart failure (PEP-CHF) study. Eur Heart $J$ 2006; 27: 2338-2345.

13. Pitt B, Pfeffer MA, Assmann SF, et al.; TOPCAT Investigators. Spironolactone for heart failure with preserved ejection fraction. $N$ Engl J Med 2014; 370: 1383-1392.

14. Komajda M, Isnard R, Cohen-Solal A, et al.; prEserveD left ventricular ejectIon fraction chronic heart Failure with ivabradine studY (EDIFY) Investigators. Effect of ivabradine in patients with heart failure with preserved ejection fraction: The EDIFY randomized placebocontrolled trial. Eur J Heart Fail 2017; 19: 1495-1503.

15. Böhm M, Drexler H, Oswald H, et al.; OptiLink HF Study Investigators. Fluid status telemedicine alerts for heart failure: A randomized controlled trial. Eur Heart $J$ 2016; 37: 3154-3163.

16. Domingo M, Lupón J, González B, et al. Evaluation of a telemedicine system for heart failure patients: Feasibility, acceptance rate, satisfaction and changes in patient behavior: Results from the CARME (CAtalan Remote Management Evaluation) study. Eur J Cardiovasc Nurs 2012; 11: 410-418.

17. Comín-Colet J, Enjuanes C, Verdú-Rotellar JM, et al. Impact on clinical events and healthcare costs of adding telemedicine to multidisciplinary disease management programmes for heart failure: Results of a randomized controlled trial. J Telemed Telecare 2016; 22: 282-295.

18. Inglis SC, Clark RA, Dierckx R, et al. Structured telephone support or non-invasive telemonitoring for patients with heart failure. Heart 2017; 103: 255-257.

19. Anker SD, Koehler F and Abraham WT. Telemedicine and remote management of patients with heart failure. Lancet 2011; 378: 731-739.

20. Chaudhry SI, Mattera JA, Curtis JP, et al. Telemonitoring in patients with heart failure. $N$ Engl $J$ Med 2010; 363: 2301-2309.

21. Cleland JG, Pellicori $P$ and Dierckx R. Clinical trials in patients with heart failure and preserved left ventricular ejection fraction. Heart Fail Clin 2014; 10: 511-523.

22. Comin-Colet J, Verdú-Rotellar JM, Vela E, et al.; Working Group of the Integrated Program for Heart Failure Management of the Barcelona Litoral Mar Integrated Health Care Area, Spain. Efficacy of an integrated hospital-primary care program for heart failure: A population-based analysis of 56,742 patients. Rev Esp Cardiol (Engl Ed) 2014; 67: 283-293. 